\title{
Global asymptotic stability for a class of nonlinear chemical equations
}

\author{
David F. Anderson ${ }^{1}$
}

April 21, 2022

\begin{abstract}
We consider a class of nonlinear differential equations that arises in the study of chemical reaction systems that are known to be locally asymptotically stable and prove that they are in fact globally asymptotically stable. More specifically, we will consider chemical reaction systems that are weakly reversible, have a deficiency of zero, and are equipped with mass action kinetics. We show that if for each $c \in \mathbb{R}_{>0}^{m}$ the intersection of the stoichiometric compatibility class $c+S$ with the subsets on the boundary that could potentially contain equilibria, $L_{W}$, are at most discrete, then global asymptotic stability follows. Previous global stability results for the systems considered in this paper required $(c+S) \cap L_{W}=\emptyset$ for each $c \in \mathbb{R}_{>0}^{m}$, and so this paper can be viewed as an extension of those works.
\end{abstract}

\section{Introduction}

This paper is motivated by the consideration of a class of nonlinear systems that arises in the study of chemistry and biochemistry. Suppose there are $m$ chemical species, $\left\{X_{1}, \ldots, X_{m}\right\}$, undergoing a series of chemical reactions. For a given reaction, denote by $y, y^{\prime} \in \mathbb{Z}_{\geq 0}^{m}$ the vectors representing the number of molecules of each species consumed and created in one instance of that reaction, respectively. Using a slight abuse of notation, we associate each such $y$ (and $y^{\prime}$ ) with a linear combination of the species in which the coefficient of $X_{i}$ is $y_{i}$. For example, if

\footnotetext{
${ }^{1}$ Department of Mathematics, University of Wisconsin, Madison, WI, 53706
} 
$y=[1,2,3]^{T}$ for a system consisting of three species, we associate with $y$ the linear combination $X_{1}+2 X_{2}+3 X_{3}$. Under this association, each $y$ (and $y^{\prime}$ ) is termed a complex of the system. We may now denote any reaction by the notation $y \rightarrow y^{\prime}$, where $y$ is the source, or reactant, complex and $y^{\prime}$ is the product complex. We note that each complex will typically appear as both a source complex and a product complex in the system. Let $\mathcal{S}=\left\{X_{i}\right\}, \mathcal{C}=\{y\}$, and $\mathcal{R}=\left\{y \rightarrow y^{\prime}\right\}$ denote the sets of species, complexes, and reactions, respectively. Denote the concentration vector of the species as $x \in \mathbb{R}^{m}$. In order to know how the state of the system is changing, we need to know the rate at which each reaction is taking place. Therefore, for each reaction, $y \rightarrow y^{\prime}$, there is a $C^{1}$ function $R_{y \rightarrow y^{\prime}}(\cdot)$ satisfying the following:

1. $R_{y \rightarrow y^{\prime}}(\cdot)$ is a function of the concentrations of those species contained in the source complex, $y$.

2. $R_{y \rightarrow y^{\prime}}(\cdot)$ is monotone increasing in each of its inputs and $R_{y \rightarrow y^{\prime}}(x)=0$ if any of its inputs are zero.

The dynamics of the system are then given by

$$
\dot{x}(t)=\sum_{y \rightarrow y^{\prime} \in \mathcal{R}} R_{y \rightarrow y^{\prime}}(x(t))\left(y^{\prime}-y\right) \doteq f(x(t)),
$$

where the last equality is a definition. The functions $R_{y \rightarrow y^{\prime}}$ are typically referred to as the kinetics of the system. This notation closely matches that of Feinberg, Horn, and Jackson, and it is their results that the main theorem in this paper extends ([11, 7, 9, 10, 8]).

Integrating equation (11) gives

$$
x(t)=x(0)+\sum_{y \rightarrow y^{\prime} \in \mathcal{R}}\left(\int_{0}^{t} R_{y \rightarrow y^{\prime}}(x(s)) d s\right)\left(y^{\prime}-y\right) .
$$

Therefore, $x(t)-x(0)$ remains in the linear space $S=\operatorname{Span}\left\{y^{\prime}-y\right\}_{y \rightarrow y^{\prime} \in \mathcal{R}}$ for all time. We shall refer to the space $S$ as the stoichiometric subspace of the system and refer to the sets $c+S$, for $c \in \mathbb{R}^{m}$, as stoichiometric compatibility classes, or just compatibility classes. Later we will demonstrate that trajectories with positive initial conditions remain in $\mathbb{R}_{>0}^{m}$ for all time. The sets $(c+S) \cap \mathbb{R}_{>0}^{m}$ will therefore be referred to as the positive stoichiometric compatibility classes. Given that trajectories remain in their positive stoichiometric compatibility classes for all time, 
we see that the types of questions that one should ask about these systems differ from the questions one normally asks about nonlinear systems. For example, instead of asking whether there is a unique equilibrium value to the system (1), and then asking about its stability properties, it is clearly more appropriate to ask whether there is a unique equilibrium within each positive stoichiometric compatibility class and, if so, what are its stability properties relative to its compatibility class.

The most common kinetics chosen is that of mass action kinetics. A chemical reaction system is said to have mass action kinetics if

$$
R_{y \rightarrow y^{\prime}}(x)=k_{y \rightarrow y^{\prime}} x_{1}^{y_{1}} x_{2}^{y_{2}} \cdots x_{m}^{y_{m}},
$$

for some constant $k_{y \rightarrow y^{\prime}}$. It has been shown that for many systems of the form (1) with mass action kinetics, there is within each positive stoichiometric compatibility class precisely one equilibrium and that equilibrium is locally asymptotically stable relative to its class $([11,9,8])$. In order to show that the equilibrium values are locally stable, the following Lyapunov function is used (one for each compatibility class)

$$
V(x, \bar{x})=V(x)=\sum_{i=1}^{m}\left[x_{i}\left(\ln \left(x_{i}\right)-\ln \left(\bar{x}_{i}\right)-1\right)+\bar{x}_{i}\right],
$$

where $\bar{x}$ is the unique equilibrium of a given positive stoichiometric compatibility class. It turns out that the function $V$ "almost" acts as a global Lyapunov function. That is, $V$ is non-negative for $x \in(\bar{x}+S) \cap \mathbb{R}_{>0}^{m}$, zero only at $\bar{x}$, and strictly decreases along trajectories. However, $V$ does not tend to infinity as trajectories near the boundary of $(\bar{x}+S) \cap \mathbb{R}_{>0}^{m}$, and without such unboundedness one can not, in general, conclude global stability. It has been shown in numerous papers, however, that global stability of $\bar{x}$ does hold if there are no equilibria on the boundary of $(\bar{x}+S) \cap \mathbb{R}_{>0}^{m}([14,2,12,13])$. Therefore, work has been done giving sufficient conditions for the non-existence of boundary equilibria in order to conclude that the equilibrium value within each compatibility class is globally stable relative to its class $([2,12,13])$.

To each subset $W$ of the set of species, the set of boundary points $L_{W}$ is defined to be

$$
L_{W}=\left\{x \in \mathbb{R}^{m}: x_{i}=0 \Leftrightarrow X_{i} \in W\right\} .
$$

We will show that there are no boundary equilibria if and only if

$$
\left[(c+S) \cap \mathbb{R}_{\geq 0}^{m}\right] \cap L_{W}=\emptyset,
$$


for all $c \in \mathbb{R}_{>0}^{m}$ and for certain subsets of the species, $W$. We will then prove that global stability holds if the intersection given in equation (5) is either empty or discrete for each $c \in \mathbb{R}_{>0}^{m}$ and those same subsets, $W$. This will imply that global stability holds even if there are boundary equilibria, so long as the boundary equilibria are extreme points of the positive stoichiometric compatibility classes. To the best of our knowledge there is only one other result concerning the global stability of mass action systems with boundary equilibria, and is contained within the Ph.D. thesis of Madalena Chavez ([6]). In order to guarantee global stability even if there exist boundary equilibria, she requires that each boundary equilibrium be hyperbolic with respect to its stoichiometric compatibility class and she requires another (more technical) condition on the stable subspaces of each boundary equilibrium (see [6], pg. 106 for details). As our result are applicable to systems with boundary equilibria that are non-hyperbolic, our results can be viewed as an extension of those in [6].

The layout of the paper is as follows. In Section 2 we will introduce the systems we consider in this paper: weakly reversible, deficiency zero systems with mass action kinetics. We will then present some preliminary results and will conclude with a proof that global stability follows if there are no equilibria on the boundary of the positive stoichiometric compatibility classes. No originality is claimed for this result as it is known. Also in Section 2 we demonstrate how the "no boundary equilibria" assumption is equivalent to equation (5) holding for all $c \in \mathbb{R}_{>0}^{m}$ and certain subsets of the species, $W$. In Section 3 we extend the previous theorems to prove that global stability still holds if the intersection given in equation (5) is always either empty or discrete for those same subsets, $W$. We also show in Section 3 how the hypothesis that the intersection in equation (5) is always empty or discrete is equivalent to a condition on the extreme points of the non-negative stoichiometric compatibility classes. In Section 4 we demonstrate our results on a number of examples. Finally, in Section 5 we sketch how to extend our results to systems with non-mass action kinetics.

\section{Preliminary results}

We start with definitions taken from [11], [8], and [9].

Definition 2.1. A chemical reaction network, $\{\mathcal{S}, \mathcal{C}, \mathcal{R}\}$, is called weakly reversible if for any reaction $y \rightarrow y^{\prime}$, there is a sequence of directed reactions beginning with $y^{\prime}$ and ending with $y$. That is, there exists complexes $y_{1}, \ldots, y_{k}$ such that the following reactions are in $\mathcal{R}: y^{\prime} \rightarrow y_{1}, y_{1} \rightarrow y_{2}, \ldots, y_{k} \rightarrow y$. 
To each reaction system, $\{\mathcal{S}, \mathcal{C}, \mathcal{R}\}$, there is a unique, directed graph constructed in the following manner. The nodes of the graph are the complexes, $\mathcal{C}$. A directed edge is then placed from complex $y$ to complex $y^{\prime}$ if and only if $y \rightarrow y^{\prime} \in \mathcal{R}$. Each connected component of the resulting graph is termed a linkage class of the graph. We denote the number of linkage classes by $l$.

As shown in the introduction, each trajectory remains in its stoichiometric compatibility class for all time. There is another restriction on the trajectories of solutions to equation (1) that is given in the following lemma. The proof can be found in both [14] and [1].

Lemma 2.2. Let $x(t)$ be a solution to (1) with initial condition $x(0) \in \mathbb{R}_{>0}^{m}$. Then, $x(t) \in \mathbb{R}_{>0}^{m}$, for all $t>0$.

\subsection{Persistence and $\omega$-limit points}

By Lemma 2.2, each trajectory must remain within $\mathbb{R}_{>0}^{m}$ if its initial condition is in $\mathbb{R}_{>0}^{m}$, therefore the linear subsets of interest are the intersections of the stoichiometric compatibility classes and $\mathbb{R}_{>0}^{m}$. Recall that in the Introduction these sets were termed the positive stoichiometric compatibility classes. This paper will mainly be concerned with showing that trajectories to systems given by equation (1) remain away from the boundary of the positive stoichiometric compatibility classes. That is, we will show that the systems are persistent. To be precise, let $\phi(t, \xi)$ be the solution to equation (1) with initial condition $\xi \in \mathbb{R}_{>0}^{m}$. The set of $\omega$-limit points of the trajectory is

$$
\omega(\xi) \doteq\left\{x \in \mathbb{R}_{\geq 0}^{m}: \phi\left(t_{n}, \xi\right) \rightarrow x, \text { for some } t_{n} \rightarrow \infty\right\}
$$

Definition 2.3. A system is persistent if $\omega(\xi) \cap \partial \mathbb{R}_{>0}^{m}=\emptyset$, for each $\xi \in \mathbb{R}_{>0}^{m}$.

We refer the reader to [4, 3, 5, 15] for some of the history and usage of the notion of persistence in the study of dynamical systems. In order to show that a chemical system is persistent, it is critical to understand which points on the boundary are capable of being $\omega$-limit points. With that in mind, we introduce the following definition.

Definition 2.4. A nonempty subset $W$ of the set of species is called a semi-locking set if for each reaction in which there is an element of $W$ in the product complex, there is an element of $W$ in the reactant complex. $W$ is called a locking set if every reactant complex contains an element of $W$. 
Locking and semi-locking sets are easily understood. Suppose that $W \subset$ $\left\{X_{1}, \ldots, X_{m}\right\}$ is a locking set. Then, because every reactant complex contains an element of $W$, if the concentration of each element of $W$ is zero, each kinetic function, $R_{y \rightarrow y^{\prime}}$, must equal zero. Therefore, all of the fluxes are zero, and $\dot{x}(t)=0$. We therefore see that the system is "locked" in place. Now suppose $W$ is a semi-locking set. If the concentration of each element of $W$ is zero, then any flux which affects the species of $W$ is turned off and the elements of $W$ are "locked" at zero. Semi-locking sets have another, important, interpretation in terms of the linkage classes and weak reversibility. If the concentrations of the elements of a semi-locking set are equal to zero and the system is weakly reversible, then all of the fluxes of any linkage class with a complex containing an element of $W$ are equal to zero (and so these linkage classes are "locked"), while the fluxes of the other linkage classes are all not equal to zero. Therefore, the concept of a semi-locking set and a locking set are equivalent for systems that are weakly reversible and have only one linkage class. We note that our notion of a semilocking set is analogous to the concept of a siphon in the theory of Petri nets. See [2] for a full discussion, including historical references, of the role of Petri nets in the study of chemical reaction networks.

The following Theorem now characterizes the boundary points that have the capability of being $\omega$-limit points of the system. This result was first proved in [2], however the proof given here is completely different and straightforward.

Theorem 2.5. Let $W$ be a non-empty subset of the species. If there exists a $\xi \in$ $\mathbb{R}_{>0}^{m}$ such that $\omega(\xi) \cap L_{W} \neq \emptyset$, then $W$ is a semi-locking set.

Proof. Suppose, in order to find a contradiction, that there is a $\xi \in \mathbb{R}_{>0}^{m}$ and a subset of the species, $W$, such that $\omega(\xi) \cap L_{W} \neq \emptyset$ and $W$ is not a semi-locking set. Let $y \in \omega(\xi) \cap L_{W}$. We note that there exists a species $X_{j}$, with $X_{j} \in W$, such that at least one input to $X_{j}$ (term in $f_{j}$ of equation (1) with a positive coefficient) is non-zero if the concentrations are given by $y$, for otherwise $W$ would be a semilocking set. Therefore, because all outputs from species $X_{j}$ (terms in $f_{j}$ with a negative coefficient) are zero at $y$, there exists $\epsilon>0$ and $k>0$ such that if $x(t) \in \mathbb{R}_{>0}^{m} \cap B_{\epsilon}(y)$, then

$$
f_{j}(x(t))=x_{j}^{\prime}(t)>k,
$$

where $B_{\epsilon}(y)=\{x:|x-y|<\epsilon\}$.

Because $f(\cdot)$ is $C^{1}$, we have $\|f\|_{\infty, l o c}<M$ for some $M>0$, and this bound is valid in $\mathbb{R}_{>0}^{m} \cap B_{\epsilon}(y)$. Therefore, for any $0<a<b$, if $x(t) \in \mathbb{R}_{>0}^{m} \cap B_{\epsilon}(y)$ for 
$t \in(a, b)$, we have that

$$
|x(b)-x(a)|=\left|\int_{a}^{b} f(x(s)) d s\right| \leq(b-a) M .
$$

Now consider a partial trajectory starting on the boundary of $\mathbb{R}_{>0}^{m} \cap B_{\epsilon}(y)$ at time $t_{\epsilon}$, ending on the boundary of $\mathbb{R}_{>0}^{m} \cap B_{\epsilon / 2}(y)$ at time $t_{\epsilon / 2}$, and remaining within that annulus for all time in $\left(t_{\epsilon}, t_{\epsilon / 2}\right)$. Note that one such partial trajectory must exist every time we enter $\mathbb{R}_{>0}^{m} \cap B_{\epsilon / 2}(y)$, and this happens at least once by our assumption that $y \in \omega(\xi) \cap L_{W}$. By (주), $t_{\epsilon / 2}-t_{\epsilon} \geq \epsilon /(2 M)$. On the other hand, by (7), $x_{j}^{\prime}(t)>k$ for $t \in\left(t_{\epsilon}, t_{\epsilon / 2}\right)$. Therefore,

$$
\begin{aligned}
x_{j}\left(t_{\epsilon / 2}\right) & =x_{j}\left(t_{\epsilon}\right)+\int_{t_{\epsilon}}^{t_{\epsilon / 2}} x_{j}^{\prime}(s) d s \\
& \geq x_{j}\left(t_{\epsilon}\right)+\epsilon k /(2 M) \\
& \geq \epsilon k /(2 M) .
\end{aligned}
$$

Combining the above with the fact that we still have $x_{j}^{\prime}(t)>k$ on $\mathbb{R}_{>0}^{m} \cap B_{\epsilon / 2}(y)$, and we see that there can not exist times $t_{n}$ such that $x\left(t_{n}\right) \rightarrow y$, as $n \rightarrow \infty$. This is a contradiction and completes the proof.

Remark. Theorem 2.5 is a powerful tool for understanding the dynamics of chemical reaction systems. We see that in order to prove that a chemical system is persistent, it is sufficient to show that $\left[(c+S) \cap \mathbb{R}_{\geq 0}^{m}\right] \cap L_{W}=\emptyset$ for all $c \in \mathbb{R}_{>0}^{m}$ and all semi-locking sets $W$. We will show in Lemma 2.8 that for many reaction systems such a condition is equivalent to having no equilibria on the boundaries of the positive stoichiometric compatibility classes.

\subsection{Deficiency and the Deficiency Zero Theorem}

We require one more definition before we can state precisely the types of systems we consider in this paper.

Definition 2.6. The deficiency, $\delta$, of a reaction network $\{\mathcal{S}, \mathcal{C}, \mathcal{R}\}$ is given by $\delta=n-l-s$, where $n$ is the number of complexes of the system, $l$ is the number of linkage classes, and $s=\operatorname{dim} S$, the dimension of the stoichiometric subspace.

Remark. It has been shown that the deficiency of a reaction network is a nonnegative number. In fact, the deficiency is the dimension of a certain subspace associated with the system. See [9], [10], and [8] for details. 
The main types of systems considered in this paper are those with mass action kinetics, are weakly reversible, and have a deficiency of zero. The following theorem by Feinberg $([8,10])$ is the catalyst for studying such systems. The proof can be found in [8] or [10].

Theorem 2.7 (The Deficiency Zero Theorem). Consider a system of the form (1) with mass action kinetics that is weakly reversible and has a deficiency of zero. Then, within each positive stoichiometric compatibility class there is precisely one equilibrium value and it is locally asymptotically stable relative to its compatibility class.

In order to prove that the systems considered in the Deficiency Zero Theorem are locally asymptotically stable, the Lyapunov function (3) is used. It is shown that for $x \in(\bar{x}+S) \cap \mathbb{R}_{>0}^{m}$ (where $\bar{x}$ is the equilibrium guaranteed to exist by Theorem 2.7), $V(x) \geq 0$ with equality if and only if $x=\bar{x}$, and that $d V(x(t)) / d t<0$ for all trajectories with initial condition in $(\bar{x}+S) \cap \mathbb{R}_{>0}^{m}$. We will make use of these facts throughout the paper without reference, however we point the interested reader to the original works for details.

\subsection{Boundary equilibria}

The following Lemma shows that having no equilibria on the boundaries of the positive stoichiometric compatibility classes is equivalent to $\left[(c+S) \cap \mathbb{R}_{>0}^{m}\right] \cap$ $L_{W}=\emptyset$ for all $c \in \mathbb{R}_{>0}^{m}$ and semi-locking sets $W$. Following Lemma 2.8 we present a theorem pertaining to any system equipped with a globally defined Lyapunov function that does not necessarily go to infinity as $x$ goes to the boundary of the domain. We then use these results in combination with Theorem 2.5 to conclude that for weakly reversible, deficiency zero systems with mass action kinetics, having no equilibria on the boundary of the positive stoichiometric compatibility classes implies global stability of the equilibrium values given by Theorem 2.7. We again note that it is already known that global asymptotic stability follows from a lack of boundary equilibria. For example, in [14] Sontag showed that all trajectories must converge to the set of equilibria, and so a lack of boundary equilibria implies convergence to the unique equilibrium in the interior of the positive stoichiometric compatibility class. We rederive this result here because our methods put it in a larger context in which global stability is understood through the intersections given in equation (5) and because it makes clear how our results in Section 3 are truly a generalization of this fact. 
Lemma 2.8. For any chemical reaction system, the set of equilibria on the boundaries of the positive stoichiometric compatibility classes is contained in $\bigcup_{c} \bigcup_{W}[(c+$ $\left.S) \cap \mathbb{R}_{\geq 0}^{m}\right] \cap L_{W}$, where the first union is over $c \in \mathbb{R}_{>0}^{m}$ and the second union is over the semi-locking sets. Further, if there are no equilibria on the boundaries of the positive stoichiometric compatibility classes for a weakly reversible, deficiency zero system with mass action kinetics, then $\left[(c+S) \cap \mathbb{R}_{\geq 0}^{m}\right] \cap L_{W}=\emptyset$ for all $c \in \mathbb{R}_{>0}^{m}$ and semi-locking sets $W$.

Proof. Let $y$ be an equilibrium on the boundary of a positive stoichiometric compatibility class. Let $W$ be the set of species with a concentration of zero at $y$. Because each complex that contains an element of $W$ is providing zero flux, in order for $y$ to be an equilibrium value each reaction in which there is an element of $W$ in the product complex must have an element of $W$ in the reactant complex. Thus, $W$ is a semi-locking set and $y \in\left[(c+S) \cap \mathbb{R}_{\geq 0}^{m}\right] \cap L_{W}$, for some $c \in \mathbb{R}_{>0}^{m}$.

In order to prove the second part of the Lemma, we suppose $W$ is a semilocking set for the system and suppose $y \in\left[(c+S) \cap \mathbb{R}_{\geq 0}^{m}\right] \cap L_{W}$ for some $c \in \mathbb{R}_{>0}^{m}$. We will now produce an equilibrium value on the boundary of $(c+S) \cap \mathbb{R}_{>0}^{m}$. If $W=\mathcal{S}, y=\overrightarrow{0}$, and, because $W$ is a semi-locking set, $y$ is an equilibrium. Otherwise, consider the system consisting only of those species not in the semilocking set $W$. By the arguments in [10], the linkage classes not "locked" by $W$ form their own weakly reversible, deficiency zero system. Therefore, there is an equilibrium for that reduced system, $\bar{z}$. Let $\bar{y}=(\bar{z}, \overrightarrow{0})$ (where we have potentially rearranged the ordering of the species so that those not in the semi-locking set came first). $\bar{y}$ is a boundary equilibrium value to our original system. Therefore, the result is shown.

Theorem 2.9. Let $x(t)=x(t, x(0))$ be the solution to $\dot{x}=f(x)$ with initial condition $x(0)$, where $f$ is $C^{1}$ and the domain of definition of the system is the open set $C \subset \mathbb{R}^{m}$. Let $\bar{x} \in C$ be the unique equilibrium value to the system. Finally, suppose that there is a globally defined Lyapunov function $V$ that satisfies:

1. $V(x) \geq 0$ with equality if and only if $x=\bar{x}$.

2. $d V(x(t)) / d t \leq 0$ with equality if and only if $x(t)=\bar{x}$.

3. $V(x) \rightarrow \infty$, as $|x| \rightarrow \infty$.

Then either $x(t) \rightarrow \bar{x}$ or $x(t) \rightarrow \partial C$. 
Proof. Suppose that $x(t) \nrightarrow \bar{x}$. Because $V(\cdot)$ decreases along trajectories, the value $V(x(t))$ is bounded above by $V(x(0))$ for all $t>0$. Therefore, because $V(x) \rightarrow \infty$ as $|x| \rightarrow \infty, x(t)$ remains bounded for all $t>0$. Also, the local asymptotic stability of $\bar{x}$ combined with the fact that $x(t) \nrightarrow \bar{x}$ implies there is a $\rho>0$ such that $|x(t)-\bar{x}|>\rho$ for all $t>0$.

Let $\epsilon>0$ and for $x \in C$ let $d(x, \partial C)$ represent the distance from $x$ to the boundary of $C$. Let $C_{\epsilon}=\{x \in C \mid d(x, \partial C) \geq \epsilon$ and $|x-\bar{x}| \geq \rho\}$. Using that trajectories remain bounded for all time, we may use the continuity of the functions $\nabla V$ and $f$ to conclude that there is a positive number $\eta=\eta(\epsilon)$ such that $\nabla V(x) \cdot f(x)<-\eta$ for all $x \in C_{\epsilon}$. Therefore, the maximum amount of time that any trajectory can spend in the set $C_{\epsilon}$ is $V(x(0)) / \eta$ (for, otherwise, $x(t) \rightarrow \bar{x}$ ). Because $\epsilon>0$ was arbitrary we see that $x(t) \rightarrow \partial C$.

Corollary 2.10. If there are no equilibria on the boundaries of the positive stoichiometric compatibility classes for a weakly reversible deficiency zero system with mass action kinetics, then the unique positive equilibrium value within each positive stoichiometric compatibility class is globally asymptotically stable relative to its compatibility class.

Proof. This is a direct result of Theorems 2.5 and 2.9 and Lemma 2.8 .

\section{Main results}

By Lemma 2.8, we see that the no boundary equilibria assumption for weakly reversible deficiency zero systems with mass action kinetics is equivalent to the assumption that $\left[(c+S) \cap \mathbb{R}_{\geq 0}^{m}\right] \cap L_{W}=\emptyset$, for all $c \in \mathbb{R}_{>0}^{m}$ and all semi-locking sets $W$. This then implies global stability by Corollary 2.10, We will extend these results by proving that global stability holds if $\left[(c+S) \cap \mathbb{R}_{\geq 0}^{m}\right] \cap L_{W}$ is empty or discrete for each $c \in \mathbb{R}_{>0}^{m}$ and each semi-locking set $W$. The following definition is necessary.

Definition 3.1. For a vector $x \in \mathbb{R}^{m}$, the support of $x$, denoted $\operatorname{supp}(x)$, is the subset of the species such that $X_{i} \in \operatorname{supp}(x)$ if and only if $x_{i} \neq 0$.

Proposition 3.2. Let $\{\mathcal{S}, \mathcal{C}, \mathcal{R}\}$ be a weakly reversible, deficiency zero, mass action chemical reaction system with dynamics given by equation (1). Suppose that $y \in \omega(x(0))$ for some $x(0) \in \mathbb{R}_{>0}^{m}$. Then there must exist a nonzero $z_{0} \in S$ with $\operatorname{supp}\left(z_{0}\right) \subset \operatorname{supp}(y)$. 
Proof. If $y \in \mathbb{R}_{>0}^{m}$, there is nothing to show. Therefore, assume that $y$ is on the boundary of the positive stoichiometric compatibility class. By Theorem 2.5 , there is a semi-locking set $W$ such that $y \in\left[(x(0)+S) \cap \mathbb{R}_{\geq 0}^{m}\right] \cap L_{W}$.

Let $V(x): \mathbb{R}_{>0}^{m} \rightarrow \mathbb{R}$, be given by equation (3) and let

$$
V_{i}\left(x_{i}\right)=x_{i}\left(\ln \left(x_{i}\right)-\ln \left(\bar{x}_{i}\right)-1\right)+\bar{x}_{i}
$$

so that $V(x)=\sum_{i=1}^{m} V_{i}\left(x_{i}\right)$. Reordering the species if necessary, we suppose $W=\left\{X_{1}, \ldots, X_{d}\right\}$. Choose $\rho>0$ so small that for each $i \leq d, x_{i}<\rho \Longrightarrow$ $\ln \left(x_{i}\right)-\ln \left(\bar{x}_{i}\right)<0$. Let $\epsilon>0$ satisfy $\epsilon<\rho$. Let $t_{\epsilon}$ be a time such that $x_{i}\left(t_{\epsilon}\right) \leq \epsilon$ for all $i \leq d$ and $\left|x_{j}\left(t_{\epsilon}\right)-y_{j}\right|<\epsilon$ for all $j \geq d+1$. Let $T_{\epsilon}=\min \left\{t>t_{\epsilon}\right.$ : $\left|x_{i}(t)-y_{i}\right| \leq x_{i}\left(t_{\epsilon}\right) / 2$, for all $\left.i \leq m\right\}$. We know such $t_{\epsilon}$ and $T_{\epsilon}$ exist because $y$ is an $\omega$-limit point of the system. Note that $T_{\epsilon}>t_{\epsilon}$ and that for each $i \leq d$, $x_{i}\left(T_{\epsilon}\right)<x_{i}\left(t_{\epsilon}\right)$. We consider how $V(x(t))$ changes from time $t_{\epsilon}$ to time $T_{\epsilon}$. Applying the mean value theorem to each $V_{i}(\cdot)$ term gives

$$
\begin{aligned}
V\left(x\left(T_{\epsilon}\right)\right)-V\left(x\left(t_{\epsilon}\right)\right)= & \sum_{i=1}^{m} V_{i}\left(x_{i}\left(T_{\epsilon}\right)\right)-V_{i}\left(x_{i}\left(t_{\epsilon}\right)\right) \\
= & \sum_{i=1}^{d}\left(\ln \left(\tilde{x}_{i}\right)-\ln \left(\bar{x}_{i}\right)\right)\left(x_{i}\left(T_{\epsilon}\right)-x_{i}\left(t_{\epsilon}\right)\right) \\
& +\sum_{i=d+1}^{m}\left(\ln \left(\tilde{x}_{i}\right)-\ln \left(\bar{x}_{i}\right)\right)\left(x_{i}\left(T_{\epsilon}\right)-x_{i}\left(t_{\epsilon}\right)\right)
\end{aligned}
$$

for some $\tilde{x}_{i} \in\left[x_{i}\left(T_{\epsilon}\right), x_{i}\left(t_{\epsilon}\right)\right]$. Recalling that $V$ decreases along trajectories of $x(t)$ by Theorem 2.7, we have $V\left(x\left(T_{\epsilon}\right)\right)-V\left(x\left(t_{\epsilon}\right)\right)<0$. Note that because for $j \geq d+1$, we have $\left|\tilde{x}_{j}-y_{j}\right|<\epsilon$, there are positive constants $c_{j}$ such that $c_{j}>\left|\ln \left(\tilde{x}_{j}\right)-\ln \bar{x}_{j}\right|$, and that bound is valid for any $\epsilon<\rho$. Let $C=\sum_{j=d+1}^{m} c_{j}$.

By our choices above, we know that for each $i \in\{1, \ldots, d\}$ the following inequalities hold:

1. $\ln \left(\tilde{x}_{i}\right)-\ln \left(\bar{x}_{i}\right)<0$.

2. $x_{i}\left(T_{\epsilon}\right)-x_{i}\left(t_{\epsilon}\right)<0$.

Therefore, each piece of the first sum in equation (10) is strictly positive. Thus, to ensure that $V$ is decreasing along this trajectory, the second sum in equation (10) 
must be negative and, letting $\Delta x_{i}=x_{i}\left(T_{\epsilon}\right)-x_{i}\left(t_{\epsilon}\right)$ for each $i$, we have

$$
\begin{aligned}
\sum_{i=1}^{d}\left(\ln \left(\tilde{x}_{i}\right)-\ln \left(\bar{x}_{i}\right)\right) \Delta x_{i} & <\left|\sum_{j=d+1}^{m}\left(\ln \left(\tilde{x}_{j}\right)-\ln \left(\bar{x}_{j}\right)\right) \Delta x_{j}\right| \\
& \leq \sum_{j=d+1}^{m} c_{j}\left|\Delta x_{j}\right| .
\end{aligned}
$$

In fact, because each term on the left hand side of equation (11) is positive, a similar inequality must hold for each $i=1, \ldots, d$. That is, for $i \leq d$

$$
\left(\ln \left(\tilde{x}_{i}\right)-\ln \left(\bar{x}_{i}\right)\right) \Delta x_{i} \leq \sum_{j=d+1}^{m} c_{j}\left|\Delta x_{j}\right| .
$$

For each $i \leq d, \tilde{x}_{i} \in\left[x_{i}\left(T_{\epsilon}\right), x_{i}\left(t_{\epsilon}\right)\right]$ and $x_{i}\left(T_{\epsilon}\right), x_{i}\left(t_{\epsilon}\right)<\epsilon$. Hence, letting $\left|\ln \bar{x}_{i}\right|=k_{i}$ we have that for each $i \leq d$

$$
\left|\ln \left(\tilde{x}_{i}\right)-\ln \left(\bar{x}_{i}\right)\right| \geq|\ln \epsilon|-k_{i} .
$$

Thus, for each $i=1, \ldots, d$,

$$
\left|\Delta x_{i}\right| \leq \frac{1}{|\ln \epsilon|-k_{i}} \sum_{j=d+1}^{m} c_{j}\left|\Delta x_{j}\right| .
$$

Let $\Delta_{\max }=\sup _{j \in\{d+1, \ldots, m\}}\left\{\left|\Delta x_{j}\right|\right\}$ and $\delta(\epsilon)=\sup _{i \in\{1, \ldots, d\}}\left(|\ln \epsilon|-k_{i}\right)^{-1}$. We know $\Delta_{\max } \neq 0$ because if it were equal to zero, then the right hand side of equation (11) would be zero, which it can not be as it is strictly larger than the left hand side. Combining the above shows that for each $i=1, \ldots, d$,

$$
\left|\Delta x_{i}\right| \leq \delta(\epsilon) C \Delta_{\max }
$$

Now we consider the vector $\Delta x=x\left(T_{\epsilon}\right)-x\left(t_{\epsilon}\right) \in S$. Normalizing the vector $\Delta x$ by dividing each entry by $\Delta_{\max }$ then produces a vector $v(\epsilon) \doteq \frac{1}{\Delta_{\max }} \Delta x$ with the following properties:

1. $v(\epsilon) \in S$.

2. For $i=1, \ldots, d,\left|v_{i}(\epsilon)\right| \leq \delta(\epsilon) C$.

3. There is at least one entry in $v(\epsilon)$ with norm 1 (the one for which the maximum in the definition of $\Delta_{\max }$ was achieved), and none have a higher norm. 
4. $1 \leq|v(\epsilon)| \leq m$.

Property 4 follows from property 3. $\epsilon>0$ was arbitrary, so we may consider a sequence $\left\{\epsilon_{n}\right\}$ such that $\epsilon_{n}>\epsilon_{n+1}$ and $\epsilon_{n} \rightarrow 0$. For each $\epsilon_{n}$ we may redo the work above. This leads to a sequence of vectors $\left\{v\left(\epsilon_{n}\right)\right\}$ and a sequence of numbers $\left\{\delta\left(\epsilon_{n}\right)\right\}$ such that $\delta\left(\epsilon_{n}\right) \rightarrow 0$ and for each $n$ all four properties above hold. Because each vector from the sequence $\left\{v\left(\epsilon_{n}\right)\right\}$ is contained in the compact space $\{v: 1 \leq|v| \leq m\} \cap S$, there is a convergent subsequence $\left\{v\left(\epsilon_{n_{k}}\right)\right\}$ and a vector $z_{0}$ such that $v\left(\epsilon_{n_{k}}\right) \rightarrow z_{0} \in\{v: 1 \leq|v| \leq m\} \cap S \subset S$, as $k \rightarrow \infty$. Note that $z_{0}$ can not be the zero vector because $\left|z_{0}\right|>1$. However, $\delta\left(\epsilon_{n_{k}}\right) \rightarrow 0$, and so the first $d$ components of $z_{0}$ are equal to zero. Hence, $\operatorname{supp}\left(z_{0}\right) \subset \operatorname{supp}(y)$.

Theorem 3.3. Let $\{\mathcal{S}, \mathcal{C}, \mathcal{R}\}$ be a weakly reversible, deficiency zero, mass action chemical reaction system with dynamics given by equation (1). Suppose that for each $c \in \mathbb{R}_{>0}^{m}$ and each semi-locking set $W$, the set $\left[(c+S) \cap \mathbb{R}_{\geq 0}^{m}\right] \cap L_{W}$ is either empty or discrete. Then the unique positive equilibrium of each stoichiometric compatibility class guaranteed to exist by the Deficiency Zero Theorem is globally asymptotically stable relative to its compatibility class.

Proof. We suppose, in order to find a contradiction, that there is a positive equilibrium, $\bar{x}$, that is not globally asymptotically stable relative to its compatibility class. By Theorems 2.5, 2.7, and 2.9, there is a semi-locking set $W$, a $\xi \in \mathbb{R}_{>0}^{m}$, and a vector $y$ such that $y \in\left[(\bar{x}+S) \cap \mathbb{R}_{\geq 0}^{m}\right] \cap L_{W}$ and $y \in \omega(\xi)$. By Proposition 3.2, there exists a nonzero $z_{0} \in S \operatorname{such}$ that $\operatorname{supp}\left(z_{0}\right) \subset \operatorname{supp}(y)$. Because $y \in \bar{x}+S$ and $z_{0} \in S$, for any $\eta>0$ we have $y+\eta z_{0} \in \bar{x}+S$. Further, because $\operatorname{supp}\left(z_{0}\right) \subset \operatorname{supp}(y)$, if $\eta$ is small enough we have that $y+\eta z_{0} \in \mathbb{R}_{>0}^{m} \cap L_{W}$. But this is valid for all $\eta$ small enough, and so $\left[(\bar{x}+S) \cap \mathbb{R}_{\geq 0}^{m}\right] \cap L_{W}$ is not discrete. This is a contradiction and so the result is shown.

Corollary 3.4. Suppose that for a weakly reversible deficiency zero chemical reaction system with mass action kinetics, each semi-locking set is a locking set. Suppose further that within each stoichiometric compatibility class, the set of equilibria on the boundary is discrete. Then the unique positive equilibrium of each stoichiometric compatibility class guaranteed to exist by the Deficiency Zero Theorem is globally asymptotically stable relative to its compatibility class.

Proof. Because each semi-locking set is a locking set, the set of boundary equilibria for a given compatibility class is precisely given by $\bigcup_{W}\left[(c+S) \cap \mathbb{R}_{\geq 0}^{m}\right] \cap L_{W}$, where the union is over the set of semi-locking sets. Therefore, each $[(c+S) \cap$ $\left.\mathbb{R}_{\geq 0}^{m}\right] \cap L_{W}$ is discrete and invoking Theorem 3.3 completes the proof. 
Corollary 3.5. Suppose that a weakly reversible deficiency zero chemical reaction system with mass action kinetics has only one linkage class. Suppose further that within each stoichiometric compatibility class, the set of equilibria on the boundary is discrete. Then the unique positive equilibrium of each stoichiometric compatibility class guaranteed to exist by the Deficiency Zero Theorem is globally asymptotically stable relative to its compatibility class.

Proof. For single linkage class systems that are weakly reversible, each semilocking set is a locking set. Using Corollary 3.4 completes the proof.

\subsection{Connection with extreme points}

We connect our results to a condition on the extreme points of the positive stoichiometric compatibility classes.

Proposition 3.6. For $y \in \mathbb{R}_{\geq 0}^{m}$, let $W=\left\{X_{i}: y_{i}=0\right\}=\operatorname{supp}(y)^{C}$. Then the following are equivalent:

(i) $y$ is an extreme point of $(y+S) \cap \mathbb{R}_{\geq 0}^{m}$.

(ii) $\left[(y+S) \cap \mathbb{R}_{\geq 0}^{m}\right] \cap L_{W}=\{y\}$.

Proof. ((ii) $\Longrightarrow$ (iii) Suppose that (ii) is true, but that (iii) is not. Then, because $\left[(y+S) \cap \mathbb{R}_{\geq 0}^{m}\right] \cap L_{W}$ is not discrete, there exists a $v \in S \cap L_{W}$ such that for sufficiently small $\epsilon, y \pm \epsilon v \in\left[(y+S) \cap \mathbb{R}_{\geq 0}^{m}\right] \cap L_{W}$. Noting that $y=(1 / 2)(y+$ $\epsilon v)+(1 / 2)(y-\epsilon v)$ then shows $y$ is not an extreme point of $(y+S) \cap \mathbb{R}_{\geq 0}^{m}$, which is a contradiction.

((ii) $\Longrightarrow$ (ii)) Suppose that (ii) is true, but that (ii) is not. Because $y$ is not an extreme point of $(y+S) \cap \mathbb{R}_{\geq 0}^{m}$, there exists nonzero vectors $v_{1} \neq y, v_{2} \neq y$ in $(y+S) \cap \mathbb{R}_{\geq 0}^{m}$ and $0<\lambda<1$ such that

$$
y=\lambda v_{1}+(1-\lambda) v_{2} .
$$

Because $v_{1}, v_{2} \in(y+S) \cap \mathbb{R}_{\geq 0}^{m}$, there exists $u, w \in S$ such that $v_{1}=y+u$ and $v_{2}=y+w$, and $u_{i}, w_{i} \geq 0$ if $X_{i} \in W$. However, because $\lambda, 1-\lambda>0$, we see by equation (12) that $u_{i}, w_{i}=0$ for all $X_{i} \in W$. Therefore, $v_{1}, v_{2} \in$ $\left[(y+S) \cap \mathbb{R}_{\geq 0}^{m}\right] \cap L_{W}$, contradicting (iii).

Theorem 3.3 can now be reformulated in the following way. 
Theorem 3.7. Let $\{\mathcal{S}, \mathcal{C}, \mathcal{R}\}$ be a weakly reversible, deficiency zero, mass action chemical reaction system with dynamics given by equation (1). For any boundary point $y \in\left[(c+S) \cap \mathbb{R}_{\geq 0}^{m}\right]$ of a positive stoichiometric compatibility class, let $W_{y}=$ $\left\{X_{i}: y_{i}=0\right\}=\operatorname{supp}(y)^{C}$. Finally, suppose that $W_{y}$ is a semi-locking set only if $y$ is an extreme point. Then the unique positive equilibrium of each stoichiometric compatibility class guaranteed to exist by the Deficiency Zero Theorem is globally asymptotically stable relative to its compatibility class.

Proof. Suppose $W$ is a semi-locking set. Let $y \in L_{W}$ be such that there exists a $c \in \mathbb{R}_{>0}^{m}$ with $y \in c+S$. If no such $y$ and $c$ exist, then $\left[(c+S) \cap \mathbb{R}_{>0}^{m}\right] \cap L_{W}=\emptyset$ for all $c \in \mathbb{R}_{>0}^{m}$. If such a $y$ and $c$ do exist, then $W=W_{y}$ and, by assumption, $y$ is an extreme point. Thus, by Proposition 3.6, $\left[(y+S) \cap \mathbb{R}_{\geq 0}^{m}\right] \cap L_{W}=$ $\left[(c+S) \cap \mathbb{R}_{\geq 0}^{m}\right] \cap L_{W}$ is discrete. Invoking Theorem 3.3 completes the proof.

\section{Examples}

We begin with an example found in [6] for a receptor-ligand model. See [6] for full details.

Example 4.1. Consider the following system, which we assume has mass action kinetics

$$
\begin{array}{ccc}
2 A+C & \rightleftarrows & A+D \\
\uparrow \downarrow & & \uparrow \downarrow \\
B+C & \leftrightarrows & E
\end{array} .
$$

For this example there are four complexes, one linkage class, and the dimension of the stoichiometric compatibility class is easily verified to be three. Therefore, the system has a deficiency of zero and our results apply. The minimal semilocking sets (that is, those that must be contained in all others) are given by $W_{1}=$ $\{A, B, E\}, W_{2}=\{A, C, E\}$, and $W_{3}=\{C, D, E\}$. Therefore, showing that the set $\bigcup_{i=1}^{3}\left[(c+S) \cap \mathbb{R}_{\geq 0}^{5}\right] \cap L_{W_{i}}$ is discrete for any $c \in \mathbb{R}_{>0}^{5}$ would also show that the sum over all semi-locking sets is discrete. For this example, it is easily verified that

$$
S=\operatorname{Span}\left\{\left[\begin{array}{c}
1 \\
0 \\
0 \\
1 \\
-1
\end{array}\right],\left[\begin{array}{c}
0 \\
1 \\
0 \\
2 \\
-2
\end{array}\right],\left[\begin{array}{c}
0 \\
0 \\
1 \\
-2 \\
1
\end{array}\right]\right\}
$$


One method to show that for a given $i$ the set $\left[(c+S) \cap \mathbb{R}_{\geq 0}^{5}\right] \cap L_{W_{i}}$ is at most discrete is to demonstrate that there are no non-zero vectors contained in $S$ with support given by $W_{i}^{C}$. This method bypasses the need to check whether the intersection $\left[(c+S) \cap \mathbb{R}_{>0}^{5}\right] \cap L_{W_{i}}$ is non-empty. It is easily verified that there are no non-zero vectors contained in $S$ with support given by $W_{1}^{C}$ or $W_{2}^{C}$. In [6] it is shown that for each $c \in \mathbb{R}_{>0}^{5},(c+S) \cap \mathbb{R}_{\geq 0}^{5}$ does intersect one of $L_{W_{1}}$ or $L_{W_{2}}$. Therefore, there are always equilibria on the boundary, however, by our results or those found in [6], they will not affect the global stability of the interior equilibria.

Let $U_{3}=\left\{x \in \mathbb{R}^{5} \mid \operatorname{supp}(x) \in W_{3}^{C}\right\}$. It is easy to show that $U_{3} \bigcap S=$ $\operatorname{span}\left\{[2,-1,0,0,0]^{T}\right\}$. Thus, the method used in the previous paragraph does not work. Therefore, for our results to apply, we need to verify that $\left[(c+S) \cap \mathbb{R}_{>0}^{5}\right] \cap$ $L_{W_{3}}=\emptyset$, for any $c \in \mathbb{R}_{>0}^{5}$. Because $L_{W_{3}}$ is characterized by having the last three entries equal to zero, in order to prove that $\left[(c+S) \cap \mathbb{R}_{\geq 0}^{5}\right] \cap L_{W_{3}}=\emptyset$ for any $c \in \mathbb{R}_{>0}^{5}$, it is sufficient to show that the space spanned by the last three entries of the vectors in (14) does not contain a vector with strictly negative components.

We have

$$
\operatorname{Span}\left\{\left[\begin{array}{c}
0 \\
1 \\
-1
\end{array}\right],\left[\begin{array}{c}
0 \\
2 \\
-2
\end{array}\right],\left[\begin{array}{c}
1 \\
-2 \\
1
\end{array}\right]\right\}=\operatorname{Span}\left\{\left[\begin{array}{c}
0 \\
-1 \\
1
\end{array}\right],\left[\begin{array}{c}
1 \\
-1 \\
0
\end{array}\right]\right\},
$$

which does not include a strictly negative vector. Thus, $\left[(c+S) \cap \mathbb{R}_{\geq 0}^{5}\right] \cap L_{W_{3}}=\emptyset$ for any $c \in \mathbb{R}_{>0}^{5}$. Combining all of the above with Theorem 3.3 shows that for any choice of rate constants and initial condition, the system (13) has a globally asymptotically stable equilibrium value.

Example 4.2. Consider the system

$$
2 A \rightleftarrows A+B \rightleftarrows B+C .
$$

There are three complexes, one linkage class, and the dimension of the stoichiometric compatibility class is two. Therefore, the system (16) has a deficiency of zero. The minimal semi-locking sets are $W_{1}=\{A, B\}$ and $W_{2}=\{A, C\}$. The stoichiometric subspace is of dimension two and the quantity $A+B+C$ is conserved. Thus, each stoichiometric compatibility class is a plane that intersects each of $L_{W_{1}}=\left\{v: v_{1}=v_{2}=0, v_{3} \neq 0\right\}$ and $L_{W_{2}}=\left\{v: v_{1}=v_{3}=0, v_{2} \neq 0\right\}$ in precisely one point. See Figure 4.1. Therefore, by Theorem 3.3, for any choice of rate constants and initial condition, the system (16) has a globally asymptotically stable equilibrium value. We note that it is easily verified that the eigenvalues of the linearized problem around the equilibria associated with the semi-locking set $W_{1}$ are all zero, and so the results of [6] do not apply here. 


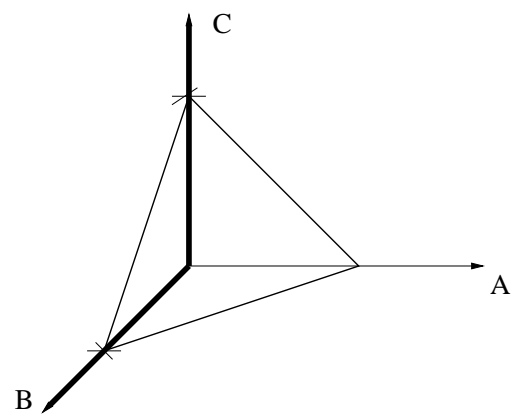

Figure 4.1: The stoichiometric compatibility class for the system (16) is a plane defined by the conservation law $A+B+C=M$ for some $M>0$. This plane intersects the sets $L_{W_{1}}$ and $L_{W_{2}}$ (bolded axes) each in precisely one point.

Example 4.3. Consider the system

$$
2 A \rightleftarrows A+B \quad 2 B \rightleftarrows A+C
$$

There are four complexes, two linkage classes, and the dimension of the stoichiometric compatibility class is two. Therefore, the system (17) has a deficiency of zero. The only minimal semi-locking set is $W=\{A, B\}$, and this is also a locking set. The stoichiometric subspace is of dimension two and the quantity $A+B+C$ is conserved. Thus, each stoichiometric compatibility class is a plane that intersects $L_{W}=\left\{v: v_{1}=v_{2}=0, v_{3} \neq 0\right\}$ in precisely one point. Therefore by Theorem 3.3 or Corollary 3.4 for any choice of rate constants and initial condition, the system (17) has a globally asymptotically stable equilibrium value. It is easily verified that the boundary equilibria are not hyperbolic with respect to their compatibility classes, and so the results of [6] do not apply in this case.

\section{Non-mass action kinetics}

In [14], Sontag extended the Deficiency Zero Theorem to systems with non-mass action kinetics. He considered weakly reversible, deficiency zero system whose kinetic functions are given by

$$
R_{y \rightarrow y^{\prime}}(x)=k_{y \rightarrow y^{\prime}} \theta\left(x_{1}\right)^{y_{1}} \cdots \theta\left(x_{m}\right)^{y_{m}},
$$

where the functions $\theta_{i}: \mathbb{R} \rightarrow[0, \infty)$ satisfy the following: 
1. Each $\theta_{i}$ is locally Lipschitz.

2. $\theta_{i}(0)=0$.

3. $\int_{0}^{1} \mid \ln \left(\theta_{i}(y) \mid d y<\infty\right.$.

4. The restriction of $\theta_{i}$ to $\mathbb{R}_{\geq 0}$ is strictly increasing and onto $\mathbb{R}_{\geq 0}$.

To prove the local asymptotic stability of the unique equilibrium within each stoichiometric compatibility class the following Lyapunov function was used

$$
V(x)=\sum_{i=1}^{m} \int_{\bar{x}_{i}}^{x_{i}}\left(\rho_{i}(s)-\rho_{i}\left(\bar{x}_{i}\right)\right) d s,
$$

where $\rho_{i}(s)=\ln \theta_{i}(s)$ and $\bar{x}$ is the unique equilibrium within the positive stoichiometric compatibility class. Note that $\theta(x)=|x|$ gives mass action kinetics, in which case the Lyapunov function given in equation (19) is the same as that in equation (3). The only dynamical property of the Deficiency Zero Theorem used in this paper was that $\nabla V(x) \rightarrow-\infty$ as $x_{i} \rightarrow 0$. We note that for the Lyapunov function (19), we still have that property because

$$
\nabla V(x)=\sum_{i=1}^{m} \rho_{i}\left(x_{i}\right)-\rho_{i}\left(\bar{x}_{i}\right),
$$

and $\rho_{i}\left(x_{i}\right)=\ln \theta_{i}\left(x_{i}\right) \rightarrow-\infty$ as $x_{i} \rightarrow 0$ by the properties of $\theta_{i}(\cdot)$ given above. Therefore, our results in this paper, and in particular Theorem 3.3. Corollary 3.4, Corollary 3.5, and Theorem 3.7, are valid in the setting (18).

\section{Acknowledgments}

I would like to thank Gheorghe Craciun for several illuminating conversations pertaining to chemical reaction systems. I would also like to thank Martin Feinberg for pointing out the connection with extreme points. This work was done under the support of the grants NSF-DMS-0109872 and NSF-DMS-0553687. 


\section{References}

[1] D. F. Anderson, Stochastic perturbations of biochemical reaction systems, Ph.D. thesis, Duke University, 2005.

[2] David Angeli, Patrick De Leenheer, and Eduardo D. Sontag, A petri net approach to the study of persistence in chemical reaction networks, Mathematical Biosciences (2007), to appear.

[3] G. Butler, H.I. Freedman, and P. Waltman, Uniformly persistent systems, Proc. Amer. Math. Soc. 96 (1986), 425-430.

[4] G. Butler and P. Waltman, Persistence in dynamical systems, J. Diff. Equ. 63 (1986), 255-263.

[5] T.C. Card, Persistence in food webs with general interactions, Mathematical Biosciences 51 (1980), 165-174.

[6] Madalena Chavez, Observer design for a class of nonlinear systems, with applications to biochemical networks, Ph.D. thesis, Rutgers, 2003.

[7] M. Feinberg, Complex balancing in general kinetic systems, Arch. Rational Mech. Anal. 49 (1972), 187-194.

[8] _ Lectures on chemical reaction networks, Delivered at the Mathematics Research Center, Univ. Wisc.Madison. Available for download at http://www.che.eng.ohiostate.edu/ feinberg/LecturesOnReactionNetworks, 1979.

[9] _ Chemical reaction network structure and the stability of complex isothermal reactors - I. the deficiency zero and deficiency one theorems, review article 25, Chem. Eng. Sci. 42 (1987), 2229-2268.

[10] Existence and uniqueness of steady states for a class of chemical reaction networks, Arch. Rational Mech. Anal. 132 (1995), 311-370.

[11] F. J. M. Horn and R. Jackson, General mass action kinetics, Arch. Rat. Mech. Anal. 47 (1972), 81-116.

[12] D. Siegel and Y.F. Chen, Global stability of deficiency zero chemical networks, Canadian Applied Mathematics Quarterly 2 (1994), no. 3, 413-434. 
[13] D. Siegel and D. MacLean, Global stability of complex balanced mechanisms, Journal of Mathematical Chemistry 27 (2000), 89-110.

[14] Eduardo D. Sontag, Structure and stability of certain chemical networks and applications to the kinetic proofreading of $t$-cell receptor signal transduction, IEEE Trans. Auto. Cont. 46 (2001), no. 7, 1028-1047.

[15] H.R. Thieme, Uniform persistence and permanence for non-autonomous semiflows in population biology, Mathematical Biosciences 166 (2000), 173-201. 